\title{
Leapfrogging Under A Sequential Investment Strategy
}

Hyung Rok Yim, The School of Business, Hanyang University, South Korea

\begin{abstract}
A common FDI pattern observed across Korean parents investing in China is that they invest sequentially. Revoking that Korean parents are intended to achieve production efficiency in China, the economies of scale of a sequential investment strategy is relatively lower compared to a large scale one-shot investment; however, the latest production technologies can be applied to sequentially established subsidiaries, which can open a strategic pathway to leapfrog other competitors in the long run. A game model is constructed to demonstrate that as longer the Korean parents are expected to stay in China, they are better off by pursuing a sequential investment strategy. Unfortunately, this result does not mean that they can leapfrog competitors through sequential investment strategy. This can happen only when they begin with larger resource endowments. The model predicts that, under the lack of resources in establishing Chinese subsidiaries, Korean firms' leapfrogging through sequential investment strategy can occur if technology shocks occur to follow-up investments after an initial investment is done. A scenario approach is performed to prove this prediction empirically. It turns out that the firm value of those Korean parents that pursue sequential investment strategy increases the most when the longer they operate in China and when their research and development investments are higher at the same time. Also, as they stay longer in China, they are intended to make more sequential investments.
\end{abstract}

Keywords: Sequential Investments; Leapfrogging; OLS; R\&D; Production

\section{INTRODUCTION}

(D) ince bilateral diplomatic relationship was established in 1992, Korean firms have penetrated into Chinese market. It is not too much to say that Korean firms' Chinese FDI's owe a lot to cultural closeness between two countries (Boeh \& Beamish, 2015; Chan, Makino \& Isobe, 2010). They were mostly aimed at expanding the scale of productions for overcoming globalization drive. A peculiar investment pattern commonly observed across Korean firms draws out our attention; in that, a few Korean firms have established subsidiaries sequentially rather than pursuing one-shot investments only. The strategic advantage of one-shot investment is to achieve higher production efficiency, which provides a stronger foundation to compete against competitors; however, tied-in effect can cause huge opportunity costs in the long-run. For those industries characterized by short product life cycles, investing a large scale one-shot investment may not be an appropriate FDI strategy. In contrast, a sequential investment strategy cannot create the economies of scale compared to the one-shot investment, but it can construct flexible production portfolios by adopting the latest technologies available. This is a real option view; sequential investments enable to adjust the levels of investment between the distinct stages of PLC (Du, Lu \& Tao, 2012). Fundamentally, FDI has technology spillover effect and its effect is spatially diffused (Kim \& Hemmert, 2016). By this respect, the sequential investment strategy is an effective competitive strategy to earn spillover effects. Of course, learning effect can enhance the performance of sequentially established subsidiaries avoiding strong tied-in effect while expanding networking ties that are critical to Korean firms' Chinese operations (Lin \& Kwan, 2016). China has two hubs for subnational activities. Beijing is a political hub and Shanghai is an economic hub. These two hubs lead both political and economic policies and subnational geographical distance becomes to affect province-level economic performances (Murphy, Andraszewicz \& Knaus, 2016).

Reflecting these discussions, sequential investment strategy, taking advantage of regional specialization through follow-up investments, can be considered as an efficient strategy, which enables Koran firms to take advantage of real options in China. According to Song (2014), geographical proximity affects positively to the financial performance 
of Japanese parents' Chinese subsidiaries; hence, one can expect that Korean parents can add more firm values as long as their sequentially established subsidiaries are not that remotely apart.

This paper tackles three mains issues taking time value into consideration because sequential investment strategy is pursued from the long-run perspective. First, whether sequential investment strategy can generate efficient production effects to Korean parents with multi Chinese subsidiaries is scrutinized. Second, it is analyzed if they can enjoy better production effects through sequential investments as the longer they stay in China. Third, it is under curiosity if sequential investments can open a strategic pathway to leapfrog large competitors.

For this purpose, a game model is constructed to draw out theoretic predictions and it is highlighted if sequential investment strategy can be used as a competitive strategy for Korean parents with Chinese subsidiaries. In the model, the longer they are expected to stay in China, Korean parents are more likely to pursue the sequential investment strategy. Korean parents may not be able to leapfrog other competitors through sequential investments generically; however, once technology shocks occur to sequentially established Chinese subsidiaries, then they might be able to leapfrog.

These theoretic predictions are examined empirically under a scenario approach. Because sequential investments are pursued from a long-run perspective while the latest technologies are applied to lately established Chinese subsidiaries, the age of Chinese subsidiaries and parent firm-wise R\&D (research and development) investments can combinedly affect firm values as well as the frequency of follow-up investments. This paper is organized as the follows. Section 2 constructs the game model and second 3 reports the characteristics of Korean parents' Chinese sequential investments. Empirical results are contained in section 4 and section 5 summarizes concluding remarks.

\section{THE GAME MODEL}

\subsection{One-Shot Investment Strategy vs. Sequential Investment Strategy}

$i$ implements FDI (foreign direct investment) in China at $t=0$ and $j$ is $i$ 's competitor in China. $i$ can make either one-shot investment or sequential investments for establishing subsidiaries in China. Market price is characterized by an inverse demand curve given to $P=1-Q$; the larger the production is, the lower the market price will be. Once $i$ establishes its subsidiaries, they are expected to be sustained till $t=n-1$. Against $i$ 's penetration, $j$ invests a large scale one-shot investment, i.e., $Q_{0}$ at $t=0$, which is denoted as the $O Q I$ strategy of $Q_{0}$.

Given $j$ 's investment, $i$ has two different investment options. First, it can either invest a small-scale one-shot investment, i.e., $q_{0}$ only at $\mathrm{t}=0$ where $Q_{0}>q_{0}$, which is denoted as the $O q I$ strategy. Second, it can invest $q_{0}$ at $t=$ 0 and does a follow-up investment $q_{1}$ at $t=1$ sequentially, which is denoted as a sequential investment $(S I)$ strategy. By $S I$, Korean parents become to be able to respond to local competitors' technological evolution in timely fashion. Without the loss of generosity, it is assumed that neither $i$ nor $j$ considers any follow-up investment once they choose $O Q I$ and $O q I$. With a view to real option theory, investments are less likely to be done as market exits are more likely to occur frequently [7]. The net present values of $i$ 's production under $O q I$ and $S I$ are given to (1) and (2) and that of $j$ 's production under $O Q I$ is given to (3).

$$
\begin{aligned}
& \tilde{O}_{i}^{q}=\left(\frac{1-\delta^{n}}{1-\delta}\right) q_{0} \\
& \tilde{O}_{i}^{s}=\left(\frac{1-\delta^{n}}{1-\delta}\right) q_{0}+\left(\frac{\delta-\delta^{n}}{1-\delta}\right) q_{1} \\
& \tilde{O}_{j}^{Q}=\left(\frac{1-\delta^{n}}{1-\delta}\right) Q_{0}
\end{aligned}
$$

Obviously, $\tilde{O}_{j}^{Q}>\tilde{O}_{i}^{q}$ because the production effect of the large scale one-shot investment dominates that of the smallscale one-shot investment. Proposition 1 suggests that $S I$ is preferred if Korean parents are generically intended to operate their Chinese subsidiaries in a longer time span. 
Proposition 1. The longer $i$ is expected to stay in China, $i$ becomes to be inclined to pursue $\widetilde{O}_{i}^{s}$.

$$
\begin{aligned}
& \text { Proof. When } n \rightarrow n+1, \tilde{O}_{i \mid n+1}^{q}-\tilde{O}_{i \mid n}^{q}=\delta^{n} q_{0} \text { while } \tilde{O}_{i \mid n+1}^{s}-\tilde{O}_{i \mid n}^{s}==\delta^{n}\left(q_{0}+q_{1}\right)>0 \text {. Thus, }\left\{\tilde{O}_{i \mid n+1}^{s}-\right. \\
& \left.\tilde{O}_{i \mid n}^{s}\right\}>\left\{\tilde{O}_{i \mid n+1}^{q}-\tilde{O}_{i \mid n}^{q}\right\} \text {. }
\end{aligned}
$$

At a glance, one can say that the more $i$ is patient, the more $i$ is likely to pursue $S I$. However, this prediction does not hold up according to Proposition 2. In other words, $O q I$ is left as a valid investment strategy to $i$; of course, $i$ will remain as $j$ ' follower if it uses $O q I$.

Proposition 2. Even when $i$ is patient enough, $i$ would not choose SI necessarily.

Proof. $\frac{\partial \tilde{o}_{i}^{s}}{\partial \delta}=\frac{\left(1-\delta^{n}\right)}{(1-\delta)^{2}} q_{0}+\frac{\left(\delta-\delta^{n}\right)}{(1-\delta)^{2}} q_{1}-\frac{n \delta^{n}}{\delta(1-\delta)}\left(q_{0}+q_{1}\right)$. Given that $1+\cdots+\delta^{n-1}=\frac{1-\delta^{n}}{(1-\delta)}$ and $\delta+\cdots+$ $\delta^{n-1}=\frac{\delta-\delta^{n}}{(1-\delta)^{2}}$, both $\frac{1-\delta^{n}}{(1-\delta)^{2}}$ and $\frac{\delta-\delta^{n}}{(1-\delta)^{2}}$ are positive; however, $-\frac{\mathrm{n} \delta^{n}}{\delta(1-\delta)}$ is negative and so the sing of $\frac{\partial \tilde{o}_{i}^{s}}{\partial \delta}$ is not clearly determined.

Proposition 3 demonstrates that $S I$ itself cannot bring competitive advantage to $i$ as $O Q I$ has a larger production effect fundamentally. This result originates from the fact that the economies of scale from $O Q I$ outweighs the time value of $S I$. In practice, differences in initial resource capacity between $i$ and $j$ may prevent $i$ 's leapfrogging, which means that $i$ is induced to pursue $S I$ due to lack in initial resource. Nevertheless, it cannot be simply concluded that $S I$ is a less efficient competitive strategy when it comes to FDI yet.

Proposition 3. Even when i's total sum of sequential investments is identical to $j$ 's large scale one-shot investment, $j$ 's production effect dominates i's.

$$
\text { Proof. } \tilde{O}_{j}^{Q}-\tilde{O}_{i}^{s}=\left(\frac{1-\delta^{n}}{1-\delta}\right)\left(Q_{0}-q_{0}\right)-\left(\frac{\delta-\delta^{n}}{1-\delta}\right) q_{1} \text {. Given } Q_{0}=q_{0}+q_{1}, \tilde{O}_{j}^{Q}-\tilde{O}_{i}^{s}{ }_{\mid Q_{0}=q_{0}+q_{1}}=q_{1}>0 \text {. }
$$

Proposition 4 demonstrates that initial investment plays a more important role in creating the production effect of SI. Practically, this indicates that $q_{1}$ has smaller discounted value. Reflecting the accumulation of market know-how, $i$ needs to pay more attention to the initial investment under $S I$.

Proposition 4. Even when $i$ is a patient sequential investor, the marginal contribution of the initial investment is greater than that of the follow-up investment.

$$
\text { Proof. } \frac{\partial^{2} \tilde{O}_{i}^{s}}{\partial \delta \partial q_{0}}-\frac{\partial^{2} \tilde{O}_{i}^{s}}{\partial \delta \partial q_{1}}=(1-\delta)>0
$$

\subsection{Leapfrogging under Technology Shock}

The previous section reveals that $i$ may not choose $S I$ and, unfortunately, it cannot leapfrog $j$ through $S I$. Then, a naturally intriguing curiosity is whether $i$ can make it possible to leapfrog. If so, first, under which condition and, second, how $i$ can leapfrog $j$ through $S I$ needs to be analyzed. This question is similar to ask if $S I$ can produce larger production effect than $O Q I$ can in the long-run.

It is meaningless to test if $i$ can dominate $j$ under $Q_{0}<q_{0}+q_{1}$. Rather, it is necessary to analyze if $i$ can take advantage of $S I$ under $Q_{0} \geq q_{0}+q_{1}$ anyhow although Proposition 3 denies such possibility. One important acknowledgement is that $q_{1}$ is produced by the latest production technology, which can provide a strategic advantage for $i$ to enhance production efficiency. Now, it is assumed that a shock occurs to $q_{1}$ as such $Q_{0}=q_{0}+\varepsilon q_{1}$.

Then, it needs to be discussed what should be the scale for $\varepsilon$ in order to outcompete $j$. If $\varepsilon$ is greater than one, it is straightforward to seeing that $q_{0}+\varepsilon q_{1}>Q_{0}$, which makes $i$ dominate $j$ easily. Then, a fundamental issue is to 
scrutinize whether $i$ can enjoy production advantage even under $0<\varepsilon \leq 1$, i.e. $Q_{0}>q_{0}+\varepsilon q_{1}$. Because Chinese local firms become to replace Korean firms' production through technological evolutions (Yim \& Jung, 2016), Korean firms need to devote more resources to earn $\varepsilon$ as the more their internal resources are restricted. The inverse demand curve is redefined as $P=1-\tilde{O}_{i}^{s}-\tilde{O}_{j}^{Q}$ under the technology shock.

It is a common knowledge that market price is lowered as the market becomes to be matured; however, Proposition 5 shows how $i$ can circumvent such pattern when $\varepsilon$ occurs. In fact, $i$ can enjoy a higher price through $S I$ overcoming the tied-in effects of $O Q I$. Most of all, it is worthwhile mentioning that the price effect in Proposition 5 can occur even when $\varepsilon \leq 1$. Such price effect again can prolong $i$ 's market residency.

Proposition 5. SI under technology shock can increase market price even in a matured market.

$$
\begin{aligned}
& \text { Proof. Given } Q_{0}=q_{0}+\varepsilon q_{1}, \frac{\partial P}{\partial Q_{0}}=-2 \frac{\left(1-\delta^{n}\right)}{1-\delta} Q_{0}<0 \text { but } \frac{\partial P}{\partial q_{1}}=\left[\frac{\left(1-\delta^{n}\right)}{1-\delta} \varepsilon-\frac{\left(\delta-\delta^{n}\right)}{1-\delta}\right] \geq 0 \text { if } \frac{\left(\delta-\delta^{n}\right)}{\left(1-\delta^{n}\right)} \leq \varepsilon \leq 1 \\
& \text { Because } \frac{\left(\delta-\delta^{n}\right)}{\left(1-\delta^{n}\right)} \leq 1, \frac{\partial P}{\partial q_{1}}>0 \text { for any } \frac{\left(\delta-\delta^{n}\right)}{\left(1-\delta^{n}\right)} \leq \varepsilon \leq 1
\end{aligned}
$$

Proposition 6 explains how $S I$ can outperform $O Q I$, which suggests that $S I$ opens a strategic pathway to outcompete $O Q I$. Figure 1 exactly depicts how this can work. Under $Q_{0}-q_{0} \leq \frac{\left(\delta-\delta^{n}\right)}{\left(1-\delta^{n}\right)} q_{1}$ where marked as dashed lines, it is $\tilde{O}_{i}^{s} \geq$ $\tilde{O}_{j}^{Q}$. Surely, $O Q I$ becomes to dominate $S I$ if $Q_{0}-q_{0}>\frac{\left(\delta-\delta^{n}\right)}{\left(1-\delta^{n}\right)} q_{1}$. Thus, $j$ can lead $i$ as long as the scale of $j$ 's $O Q I$ dominates $i$ 's initial investment; however, $i$ can have a chance to leapfrog $j$ if it becomes $Q_{0}-q_{0} \leq \frac{\left(\delta-\delta^{n}\right)}{\left(1-\delta^{n}\right)} q_{1}$ through the technology shock. In practice, dynamic learning enhances the efficacy of local subsidiaries' access to internal resources and thus technology shocks from either headquarters or subsidiaries can be interchanged simultaneously, which expands the impact of technology shocks.

Proposition 6. In the long-run, the production effect under SI can dominate that of a large scale one-shot investment under OQI if technology shocks can fill up the scale of productions between OQI and SI.

$$
\begin{aligned}
& \text { Proof. } \widetilde{O}_{j}^{Q}-\widetilde{O}_{i}^{s}=\left\{\frac{\left(1-\delta^{n}\right)}{1-\delta} \varepsilon-\frac{\left(\delta-\delta^{n}\right)}{1-\delta}\right\} q_{1} \text {. Given } Q_{0}=q_{0}+\varepsilon q_{1}, \tilde{O}_{j}^{Q}>\tilde{O}_{i}^{s} \text { if } Q_{0}>q_{0}+\frac{\left(\delta-\delta^{n}\right)}{\left(1-\delta^{n}\right)} q_{1} \text { but } \tilde{O}_{i}^{s} \geq \\
& \widetilde{O}_{j}^{Q} \text { if } q_{0}<Q_{0} \leq q_{0}+\frac{\left(\delta-\delta^{n}\right)}{\left(1-\delta^{n}\right)} q_{1} \text { where } \frac{\left(\delta-\delta^{n}\right)}{\left(1-\delta^{n}\right)}<1 .
\end{aligned}
$$

Figure 1. The Mechanism for Small-and Medium-size Firms’ Leapfrogging

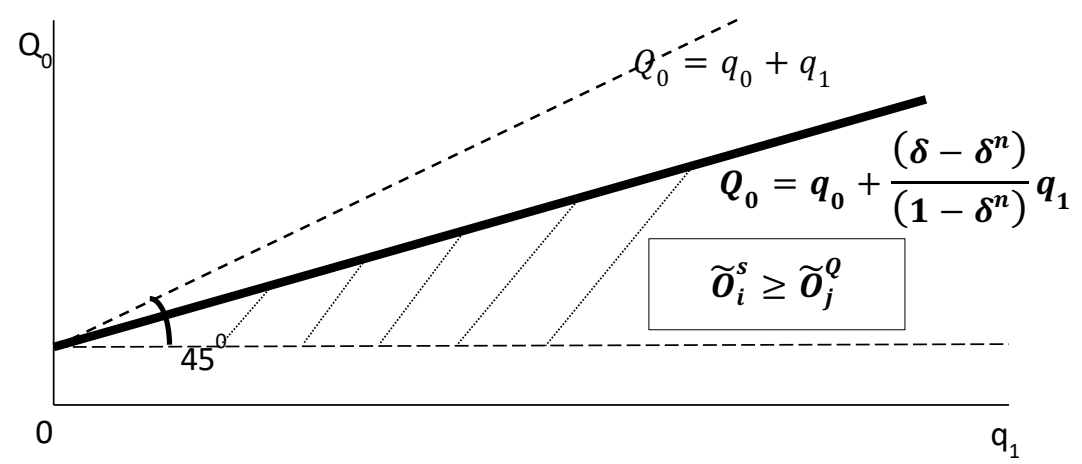




\section{EMPIRICAL WORKS}

\subsection{The Characteristics of Korean Parents' Chinese Sequential Investments}

Korean parents pursuing sequential investments in China are identified through 'Foreign Operating Korean Firms Directory 2011/12' published by KOTRA (Korea Trade-Investment Promotion Agency) as used in Zeng, Shenkar, Lee, \& Song, 2013. The book is a semi-annually published public data book and Table 1 summarizes 132 Korean parents' Chinese FDI characteristics earned from the public data book.

KOSPI listed Korean parents have, on average, 1.93 Chinese subsidiaries along to the maximum of 14. An eyecatching observation is that external audited Korean firms have more Chinese subsidiaries than KOSADQ listed parents. Furthermore, their average subsidiary size is also greater than KOSADQ listed parents. This observation reveals that small- and medium-size Korean firms have been pursuing Chinese sequential investments striving to obtain production effects. Also, Chinese government's FDI incentive policy seems to be successful as many Korean parents establish Chinese subsidiaries in SEZ (special economic zone).

Table 1. The Chinese FDI's of Korean Parents

\begin{tabular}{|c|c|c|c|c|c|c|}
\hline & Classifications & Obs. & Mean & Stand. Dev. & Min. & Max. \\
\hline \multirow{4}{*}{$\begin{array}{l}\text { The Number of Chinese } \\
\text { Subsidiaries }\end{array}$} & KOSPI & 60 & 1.93 & 2.67 & 0 & 14 \\
\hline & KOSDAQ & 26 & .58 & 1.27 & 0 & 5 \\
\hline & External Audited & 43 & .74 & 1.14 & 0 & 6 \\
\hline & Located in SEZ & 61 & 1.34 & 2.37 & 0 & 14 \\
\hline \multirow{4}{*}{ Subsidiary Size ${ }^{1}$} & KOSPI & 60 & $\$ 41.27$ & $\$ 10.01$ & $\$ 1.0$ & $\$ 678.70$ \\
\hline & KOSDAQ & 26 & $\$ 10.41$ & $\$ 10.25$ & $\$ 1.3$ & $\$ 31.0$ \\
\hline & External Audited & 43 & $\$ 15.53$ & $\$ 30.60$ & $\$ 1.0$ & $\$ 186.45$ \\
\hline & Located in SEZ & 61 & $\$ 29.15$ & $\$ 57.0$ & $\$ 1.0$ & $\$ 365.57$ \\
\hline
\end{tabular}

1. Subsidiary size is measured by million-dollar basis.

2. Source: KOTRA, Foreign Operating Korean Firms Directory 2011/12.

\subsection{Empirical Framework}

(4)-(6) are designed to examine the contributions of Korean Parents' SI to the total firm values measured by market capitalization. $y_{i}$ is natural logged market capitalization in 2012. $c$ is a constant while $c_{1 d}, c_{2 d}$, and $c_{3 d}$ are industry dummies. ${ }^{1}$ In $(4), s_{4^{-}, i}\left(s_{5^{+}, i}\right)$ is a dummy that gives the value of one to those firms that made follow-up investments within (after) four (five) years ex post its initial investment. If $s_{5^{+}, i}$ has a larger effect than $s_{4^{-}, i}$, it can be said that the latest follow-up investments contribute more to Korean parents' firm values. As explanatory variables, $s z_{i}$ represents the investment size of Korean parents' subsidiaries. $e_{i}$ is labor equipment ratio, $c i_{i}$ is capital intensity, $r i_{i}$ is R\&D intensity, $m i_{i}$ is marketing intensity, which are adjusted by $i$ 's total assets.

In (5), $s g_{d}$ represents the number of subsidiaries that are established by green field investment and $s m_{d}$ does the number of subsidiaries established by M\&A (mergers and acquisitions). From the long-run perspective, $s g_{d}$ is expected to show larger effects. (6) attempts a scenario analysis. $a g r_{d}^{h h}$ represents the case when subsidiary age measured by initial investment is older than the average subsidiary's age of the sample and when Korean firms' R\&D investments are higher than the average R\&D investment of the sample at the same time. $a g r_{d}^{h l}, a g r_{d}^{l h}$, and $a g r_{d}^{l h}$ are similarly defined but $a g r_{d}^{l h}$ is dropped to avoid dummy trap. If $a g r_{d}^{l h}$ can have larger effects compared to $a g r_{d}^{h l}$, one can say that technology shock obviously plays an important role in expanding $i$ 's total firm value. $n s_{i}$ is the number of $i$ 's subsidiaries.

${ }^{1}$ C1 Manufacturing includes Paper, textile, apparel, foods and C2 Manufacturing does electricity, electronics, machinery, steel, metal, and chemicals. C3 Manufacturing includes transportation industry and service industry does telecommunication, service, construction, education. C1, $\mathrm{C} 2, \mathrm{C} 3$, and service industries occupy $13 \%, 54 \%, 16 \%$, and $16 \%$, respectively. In order to avoid dummy trap, service industry dummy is dropped. 


$$
\begin{aligned}
& y_{i}=c+c_{1 d}+c_{2 d}+c_{3 d}+s_{4^{-}, i}+s_{5^{+}, i}+s z_{i}+e_{i}+c i_{i}+r i_{i}+m i_{i}+\varepsilon_{i} \\
& y_{i}=c+c_{1 d}+c_{2 d}+c_{3 d}+s g_{d}+s m_{d}+s z_{i}+e+c i_{i}+r i_{i}+m i_{i}+\varepsilon_{i} \\
& y_{i}=c+c_{1 d}+c_{2 d}+c_{3 d}+a g r_{d}^{h h}+a g r_{d}^{h l}+a g_{r d}^{l l}+n s_{i}+s z_{i}+e_{i}+c i_{i}+r i_{i}+m i_{i}+\varepsilon_{i}
\end{aligned}
$$

(7) can exhibit whether Korean parents pursue $S I$ when they are expected to stay longer in China. The dependent variable $a g_{i}$ is $i$ 's length of residency in China which is measured by the total years of operation through $i$ 's subsidiaries. If $n s_{i}$ shows a positive and significant coefficient, it implies that as longer the residency of Korean parents in China is, the more the follow-up investments are likely to be invested. (8) tests under which scenario Korean parents become to pursue $S I$.

$$
\begin{aligned}
& a g_{i}=c+c_{1 d}+c_{2 d}+c_{3 d}+n s_{i}+e_{i}+c i_{i}+r i_{i}+m i_{i}+\varepsilon_{i} \\
& n s_{i}=c+c_{1 d}+c_{2 d}+c_{3 d}+a g r_{d}^{h h}+a g r_{d}^{h l}+a g_{r d}^{l l}+e_{i}+c i_{i}+r i_{i}+m i_{i}+\varepsilon_{i}
\end{aligned}
$$

\section{EMPIRICAL RESULTS}

Table 2 contains the estimation results for (4)-(6). $s_{5^{+}, i}$ is estimated to be positive and significant while $s_{4^{-}, i}$ is insignificant, which suggests that those Korean parents that made follow-up investments lately can earn more from $S I$. Between green field investment and M\&A, the former can add more firm values.

The scenario analysis reveals that both $a g r_{d}^{h h}$ and $a g r_{d}^{l h}$ have positive and significant effects but $a g r_{d}^{h h}$ show a larger effect. This demonstrates that technology shock is the main driver for SI. Also, its effect becomes to be dominant as longer the Korean parents are expected to stay in China as well. 
Table 2. The Contribution of SI to Korean Parents' Firm Value

\begin{tabular}{|c|c|c|c|}
\hline \multirow{2}{*}{ Variables } & \multicolumn{3}{|c|}{ Dependent Variable: Total Market Capitalization } \\
\hline & Eq. (4) & Eq. (5) & Eq. (6) \\
\hline Constant & $\begin{array}{c}-5.3303^{* * *} \\
(1.9970)\end{array}$ & $\begin{array}{c}-4.8150^{* *} \\
(2.0264)\end{array}$ & $\begin{array}{l}-3.0585 \\
(1.9800)\end{array}$ \\
\hline$c_{1 d}$ & $\begin{array}{c}-2.3059^{* * *} \\
(0.8473)\end{array}$ & $\begin{array}{c}-2.2944^{* * *} \\
(0.8421)\end{array}$ & $\begin{array}{c}-1.9956^{* * *} \\
(0.7467)\end{array}$ \\
\hline$c_{2 d}$ & $\begin{array}{c}-2.2900^{* * *} \\
(0.7276)\end{array}$ & $\begin{array}{c}-2.2950^{* * *} \\
(0.7308)\end{array}$ & $\begin{array}{c}-1.7186^{* * *} \\
(0.5115)\end{array}$ \\
\hline$c_{3 d}$ & $\begin{array}{c}-1.5538^{* *} \\
(0.7660)\end{array}$ & $\begin{array}{c}-1.5689^{* *} \\
(0.7622)\end{array}$ & $\begin{array}{c}-0.9788 \\
(0.6120)\end{array}$ \\
\hline$S_{4^{-}, i}$ & $\begin{array}{c}0.2147 \\
(0.1442)\end{array}$ & - & - \\
\hline$S_{5^{+}, i}$ & $\begin{array}{c}0.2205^{* * *} \\
(0.0591)\end{array}$ & - & - \\
\hline$s g_{d}$ & - & $\begin{array}{c}0.2770^{* * *} \\
(0.0851)\end{array}$ & - \\
\hline$s m_{d}$ & - & $\begin{array}{c}-0.0982 \\
(0.3747)\end{array}$ & - \\
\hline$a g r_{d}^{h h}$ & - & - & $\begin{array}{c}2.8197^{* * *} \\
(0.2798)\end{array}$ \\
\hline$a g r_{d}^{h l}$ & - & - & $\begin{array}{c}0.2244 \\
(0.4003)\end{array}$ \\
\hline$a g r_{d}^{l h}$ & - & - & $\begin{array}{l}1.5622^{* * *} \\
(0.4242)\end{array}$ \\
\hline$n s_{i}$ & - & - & $\begin{array}{c}0.0968^{*} \\
(0.0565)\end{array}$ \\
\hline$s z_{i}$ & $\begin{array}{c}0.5639^{* * *} \\
(0.1260)\end{array}$ & $\begin{array}{c}0.5307^{* * *} \\
(0.1261)\end{array}$ & $\begin{array}{l}0.3674^{*} \\
(0.1290)\end{array}$ \\
\hline$e_{i}$ & $\begin{array}{c}2.6865^{* * *} \\
(0.9371)\end{array}$ & $\begin{array}{c}2.7510^{* * *} \\
(0.9018)\end{array}$ & $\begin{array}{c}2.6154^{* * *} \\
(0.5374)\end{array}$ \\
\hline$c i_{i}$ & $\begin{array}{l}-0.0046 \\
(0.0152)\end{array}$ & $\begin{array}{l}-0.0135 \\
(0.0163)\end{array}$ & $\begin{array}{c}-0.0066^{* * *} \\
(0.0121)\end{array}$ \\
\hline$r i_{i}$ & $\begin{array}{c}0.2983^{* * *} \\
(0.0944)\end{array}$ & $\begin{array}{c}0.2933^{* * *} \\
(0.0927)\end{array}$ & - \\
\hline$m i_{i}$ & $\begin{array}{c}0.0913 \\
(0.0923)\end{array}$ & $\begin{array}{c}0.0851 \\
(0.0938)\end{array}$ & $\begin{array}{c}0.1321^{*} \\
(0.0666)\end{array}$ \\
\hline$R^{2}$ & 0.5014 & 0.5122 & 0.6226 \\
\hline Obs. & 87 & 87 & 87 \\
\hline
\end{tabular}

1. For correcting heterogeneity, White standard errors are reported

2 . *, $* * * * *$ are significant at $10 \%, 5 \%$, and $1 \%$, respectively.

According to Table $3, n s_{i}$ has a positive and significant coefficient on subsidiary age, which points out that those Korean parents owning multi subsidiaries in China are likely to be those group of firms stay longer in Chia. In parallel to Table 2, both $\operatorname{agr}_{d}^{h h}$ and $a g r_{d}^{l h}$ have positive and significant effects on $n s_{i}$; the number of Korean parents' Chinese subsidiaries will be increasing more under $a g r_{d}^{h h}$. 
Table 3. The Scenario Approach on Korean Parents' Time Value

\begin{tabular}{|c|c|c|}
\hline Variables & Dependent Variable: Subsidiaries’ Age & $\begin{array}{c}\text { Dependent Variable: The Number of } \\
\text { Chinese Subsidiaries }\end{array}$ \\
\hline Constant & $\begin{array}{l}2.2136^{* * *} \\
(0.1089)\end{array}$ & $\begin{array}{c}0.4478 \\
(0.4513) \\
\end{array}$ \\
\hline$c_{1 d}$ & $\begin{array}{c}0.1342 \\
(0.1238)\end{array}$ & $\begin{array}{l}-0.3874 \\
(0.5101)\end{array}$ \\
\hline$c_{2 d}$ & $\begin{array}{c}0.0291 \\
(0.1066)\end{array}$ & $\begin{array}{l}-0.2302 \\
(0.4653)\end{array}$ \\
\hline$c_{3 d}$ & $\begin{array}{l}-0.2007 \\
(0.1630)\end{array}$ & $\begin{array}{l}-0.4160 \\
(0.4687)\end{array}$ \\
\hline$n s_{i}$ & $\begin{array}{l}0.0448^{* *} \\
(0.020)\end{array}$ & - \\
\hline$a g r_{d}^{h h}$ & - & $\begin{array}{l}2.0299^{* *} \\
(0.8566)\end{array}$ \\
\hline$a g r_{d}^{h l}$ & - & $\begin{array}{c}0.4285 \\
(0.4862)\end{array}$ \\
\hline$a g r_{d}^{l h}$ & - & $\begin{array}{l}0.5719^{*} \\
(0.2943)\end{array}$ \\
\hline$e_{i}$ & $\begin{array}{r}-0.2848 \\
(.2438)\end{array}$ & $\begin{array}{l}1.7222^{* *} \\
(0.8953)\end{array}$ \\
\hline$c i_{i}$ & $\begin{array}{c}-0.0088^{* *} \\
(.0034)\end{array}$ & $\begin{array}{l}0.1199^{* * *} \\
(0.0073)\end{array}$ \\
\hline$r i_{i}$ & $\begin{array}{c}-0.00180 \\
(.0255)\end{array}$ & - \\
\hline$m i_{i}$ & $\begin{array}{l}0.0155 \\
(.0207)\end{array}$ & $\begin{array}{l}0.0574^{* *} \\
(0.0416)\end{array}$ \\
\hline$R^{2}$ & 0.1087 & 0.2785 \\
\hline Obs. & 131 & 131 \\
\hline
\end{tabular}

1. For correcting heterogeneity, White standard errors are reported

2 . $* * *, * *$ are significant at $10 \%, 5 \%$, and $1 \%$, respectively.

\section{CONCLUSIONS}

The main theme of the paper was to explore if sequential investment strategy can provide strategic advantages to Korean parents investing in China and, if so, how such advantages can be applied in real businesses were scrutinized in several ways. According to the game model's predictions, sequential investment strategy itself does not necessarily make them lead market competition. However, once technology shocks occur, then sequentially established subsidiaries became to be able to enhance their discounted values while achieving more refined production efficiency.

This outcome provides several strategic momenta to small- and medium-size enterprises. Under one-shot game architecture, they are not able to outcompete large competitors generically due to lack in resource endowments. Technology shocks can fill up resource differences between the group of small- and medium-size firms and large firms; as a result, they can leapfrog large competitors under one-shot investment strategy even with a smaller total sum of sequential investment. The key point was turned out to be their patience because they can pursue sequential investments looking forward to earning new innovations. Another advantage is that technology shocks, combined with sequential investments, can prolong product life cycles, which can be highlighted as another critical factor to explain how leapfrogging occurs in real business area.

Therefore, it can be said that sequential investments are necessary for Korean parents to compete in China but technology shocks, that can support sequentially established subsidiaries, are sufficient to expand production effects through sequential investments. 


\section{AUTHOR BIOGRAPHY}

Hyung Rok Yim is an associate professor at the School of Business, Hanyang University.

\section{REFERENCES}

Boeh, K. K., \& Beamish, P. W. (2015). The cost of distance on subsidiary performance. Asian Business \& Management, 14(3), 171-193.

Chan, C. M., Makino, S., \& Isobe, T. (2010). Does subnational region matter? Foreign affiliate performance in the United States and China. Strategic Management Journal, 31(11), 1226-1243.

Du, J., Lu, Y., \& Tao, Z. (2012). Institutions and FDI location choice: The role of cultural distances. Journal of Asian Economics, 23(3), 210-223.

Kim, J. J., \& Hemmert, M. (2016). What drives the export performance of small and medium-sized subcontracting firms? A study of Korean manufacturers. International Business Review, 25(2), 511-521.

Lin, M., \& Kwan, Y. K. (2016). FDI technology spillovers, geography, and spatial diffusion. International Review of Economics \& Finance, 43, 257-274.

Murphy, R. O., Andraszewicz, S., \& Knaus, S. D. (2016). Real options in the laboratory: An experimental study of sequential investment decisions. Journal of Behavioral and Experimental Finance, 12, 23-39.

Song, S. (2014). Unfavorable market conditions, institutional and financial development, and exits of foreign subsidiaries. Journal of International Management, 20(3), 279-289.

Yim, H. R., \& Jung, W. (2016). Sequential Foreign Direct Investment under Information Asymmetry: Lessons from Korean companies' outward FDIs to China. Transformation in Business \& Economics, 15(3), 80-94.

Zeng, Y., Shenkar, O., Lee, S. H., \& Song, S. (2013). Cultural differences, MNE learning abilities, and the effect of experience on subsidiary mortality in a dissimilar culture: Evidence from Korean MNEs. Journal of International Business Studies, 44(1), 42-65. 


\section{NOTES}

